\title{
Optimization of finite diffraction gratings for the excitation of surface plasmons
}

\author{
Gaëtan Lévêque $^{\text {a) }}$ and Olivier J. F. Martin \\ Nanophotonics and Metrology Laboratory, ${ }^{\text {b) }}$ Swiss Federal Institute of Technology Lausanne (EPFL), \\ 1015 Lausanne, Switzerland
}

(Received 20 January 2006; accepted 6 October 2006; published online 18 December 2006)

\begin{abstract}
The excitation of a surface plasmon polariton (SPP) wave on a metal-air interface by a diffraction grating under monochromatic normal illumination is investigated numerically. The influence of the different experimental parameters (grating thickness, period, and duty cycle) is discussed in detail for a semi-infinite metal and a thin film. Both engraved (grooves) and deposited (protrusions) gratings are considered. The most efficient coupling to the SPP is obtained for a groove grating which duty cycle is about 0.5 . Furthermore a small grating depth of some tens of nanometers is sufficient to excite a SPP mode with a coupling efficiency higher than $16 \%$ in each direction. Implications for practical SPP experiments are discussed. (C) 2006 American Institute of Physics.
\end{abstract}

[DOI: $10.1063 / 1.2401025$ ]

\section{INTRODUCTION}

Surface plasmon polaritons (SPPs) are electromagnetic modes confined to the close vicinity of a metal-dielectric interface, which originate from oscillations of the electronic charge density. ${ }^{1}$ These modes propagate parallel to the interface, on a length that depends on the geometry of the system and ranges from some tens to several hundreds of micrometers. This phenomenon has a wide variety of applications, including the miniaturization of electronic and light processing devices ${ }^{2}$ and the creation of optical biochip ${ }^{3-5}$ to study complex macromolecules.

For all these applications, the efficient coupling of a macroscopic external field to the SPP is a key issue, which still remains quite challenging to realize practically. Indeed, the parallel component of the SPP wave vector is longer than that of a propagating incoming field, which therefore cannot excite the SPP. A commonly used technique to overcome this problem is based on the creation of an evanescent wave by total internal reflection of an incident field on an interface with a more refractive dielectric medium, thereby allowing to fulfill the excitation condition. ${ }^{6}$ This is usually realized in the Kreshmann configuration, where a thin metallic film deposited on a glass substrate is illuminated from the glass in total internal reflection. By conservation of the parallel component of the incoming light wave vector, the SPP can be successfully excited on the opposite metal-air interface. ${ }^{7}$

An alternative technique is the utilization of a localized object, such as the tip of a scanning near-field optical microscope or a defect deposited on the metallic surface, which diffracts the incoming field into many components both propagating and evanescent: some of these components then fulfill the excitation condition of the SPP. ${ }^{8,9}$ Moreover, a grating which period is close to the SPP wavelength allows to increase the portion of wave vectors matching that of the

\footnotetext{
a)Electronic mail: gaetan.leveque@epfl.ch

${ }^{b)}$ http://www.nanophotonis.ch/
}

SPP. However, any practical realization of such a coupling structure is finite, which raises the question of the efficiency of a small grating, composed only of a few periods. Actually, it has been shown by Ditlbacher et al. that it was possible to use only one metallic stripe deposited on a gold slab to excite the SPP. ${ }^{10,11}$

In this article, we present a numerical study of the SPP excitation using finite gratings with a small number of periods. The corresponding parameter space is very large and cannot be easily explored experimentally. The purpose of this paper is to compute the optimal grating geometry for the best energy transfer to the SPP from an external Gaussian monochromatic field at normal incidence to the surface mode. For that incidence, two SPPs are equally excited, one in the forward direction and one in the backward direction. Specifically, we study the influence of the periodicity, the height, and the width of the grating on the coupling efficiency. The calculations are performed with the dyadic Green's tensor method, based on the resolution of LippmannSchwinger equation for the electric field. ${ }^{12-14}$ It is well suited for the study of localized objects embedded in a dielectric or metallic multilayered medium, both for two-dimensional (2D) and three-dimensional (3D) geometries. Throughout the paper we consider SPP propagating on gold and use the data of Palik for its permittivity. ${ }^{15}$

The article is organized as follows: in Sec. II we first investigate the properties of SPP propagating on a flat metalvacuum interface and then the influence of an infinite grating on that interface. This section provides therefore reference solutions based on infinite systems. In Sec. III, we study how these results are modified when a finite grating with only five periods is considered. The parameters of this grating are optimized to enhance the excitation of the SPP. Two systems are studied: the first is a semi-infinite metallic space and the second is a symmetric metallic slab. Finally, concluding remarks are given in Sec. IV. 

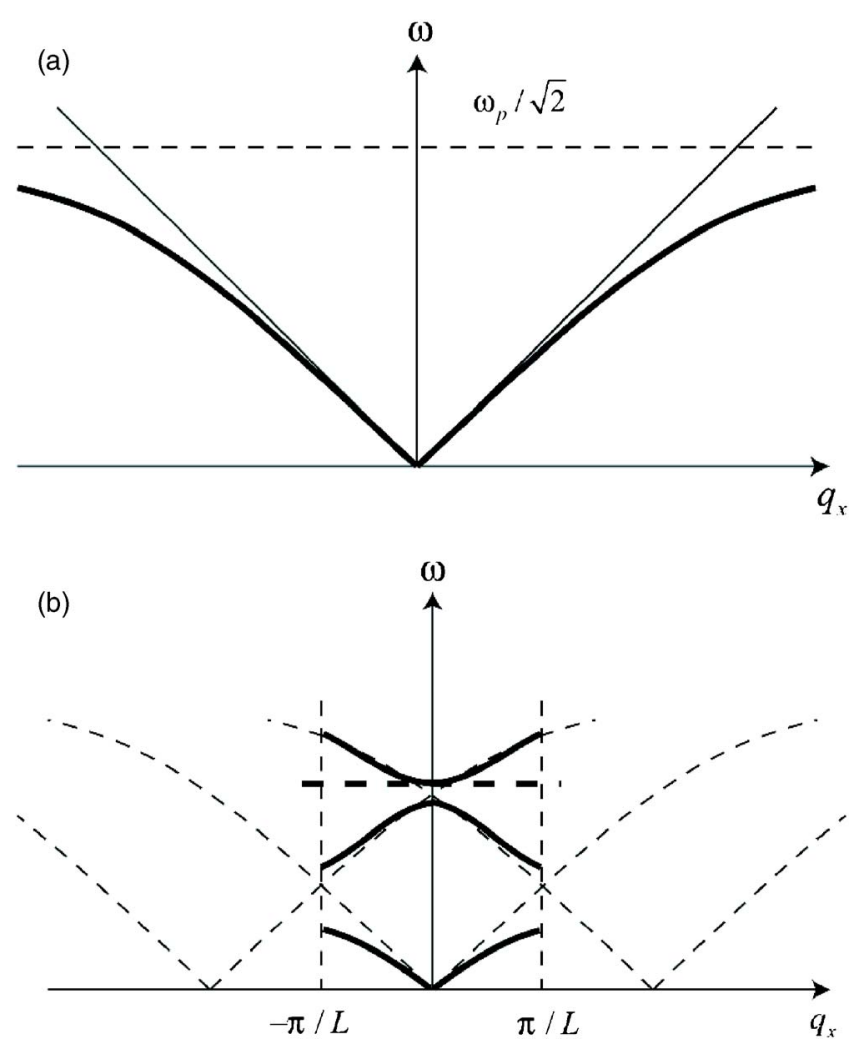

FIG. 1. (a) Dispersion curve of the SPP mode propagating at a free metal-air interface. The diagonal solid lines correspond to the light lines $\omega=|k| c$. (b) Dispersion curve of the SPP mode at a periodically modulated interface with period $L$. The modes are characterized by their frequency and their longitudinal wave vector $q_{x}$ inside the first Brillouin zone.

\section{SPP MODES OF AN INFINITE GRATING ON A METAL-VACUUM INTERFACE}

In this section we study the excitation of SPP on free and corrugated interfaces in the case of an infinite grating.

\section{A. SPP propagating on a free metal-vacuum interface}

Let us first consider the characteristics of a SPP traveling on a free metal-vacuum interface. The dispersion relation of this SPP mode reads as ${ }^{1}$

$$
q_{\mathrm{SPP}}=\frac{\omega}{c} \sqrt{\frac{\epsilon(\omega)}{\epsilon(\omega)+1}}
$$

where $q_{\mathrm{SPP}}$ is the SPP wave vector, $\omega$ the angular frequency, and $c$ the speed of light in vacuum. Equation (1) is shown in Fig. 1(a) for a Drude-like metal susceptibility $\epsilon(\omega) .{ }^{16}$

The plasmon mode cannot be excited by a propagating wave since its wave vector is always larger than the maximal value allowed for waves propagating in the vacuum, Fig. 1(a). For instance, at $\lambda=633 \mathrm{~nm}$ the SPP wavelength in this system is $596 \mathrm{~nm}$ and its propagation length is $12.1 \mu \mathrm{m}$. The propagation length is defined as the distance over which the amplitude of the SPP decreased by a factor of $e$ as it propagates. The amplitude of the SPP also decreases exponentially away from the interface inside both media. At $\lambda=633 \mathrm{~nm}$, this decay length is $280 \mathrm{~nm}$ on the vacuum side and $32 \mathrm{~nm}$ in the metal.
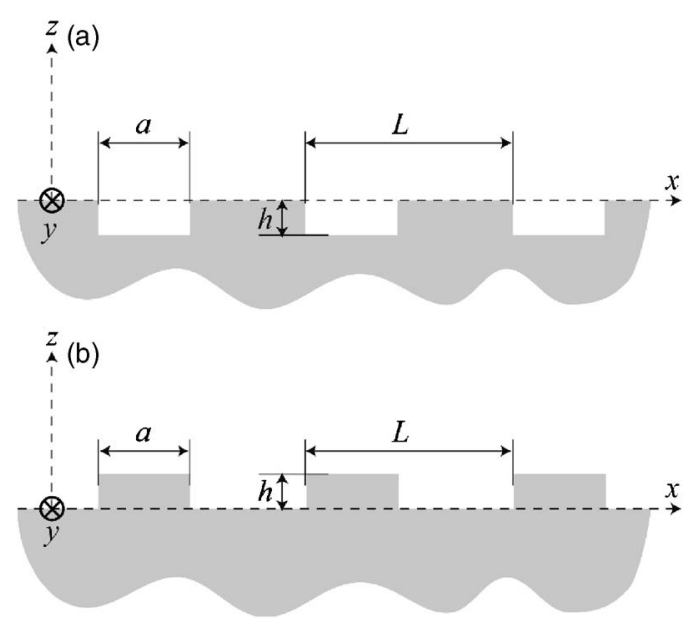

FIG. 2. (a) Geometrical parameters of a grating of gold grooves engraved in a semi-infinite gold space. The height of the modulated part is $h$, the width of the grooves is $a$, and the period is $L$. (b) For a grating of protrusions, $a$ represents the width of the metallic part.

\section{B. Modes of the corrugated interface}

Let us now consider the effect of an infinite periodic corrugation of the interface. We suppose that the corrugation is along the $x$ direction and invariant in the $y$ direction, the interface being overlaid with the $(O x y)$ plane as illustrated in Fig. 2. The plane $(0 x z)$ is both the incidence and polarization plane of the incident electric field. Figure 2(a) shows the section of a rectangular groove grating, while Fig. 2(b) shows a protrusion grating. Both structures are fully characterized by three parameters (see Fig. 2): the period $L$, the height $h$ of the modulated part, and the width $a$ of the defects [for (a) grooves and (b) protrusions in Fig. 2]. It is also convenient to define the duty cycle of the grating $\mathrm{dc}=a / L$.

When such a grating is created onto a flat interface, the SPP dispersion relation of the free metal-vacuum is modified as follows. ${ }^{17}$ In a first step, the curve is repeated periodically in the reciprocal space each $m K=m 2 \pi / L$, where $m$ is an integer and $K$ the unit Bloch vector of the grating as indicated in Fig. 1(b). The new SPP modes are then described by a wave vector inside the first Brillouin zone $[-\pi / L, \pi / L]$. At the crossing points of two curves, two diffracted waves interact, which lifts a degeneracy and forms a gap. A change of the grating shape will modify the gap width and location. As a consequence, the periodicity of the coupling grating for an incident plane wave at normal incidence $\left(q_{x}=0\right)$ does not correspond to the plasmon wavelength of the free interface. If we consider the dispersion curves in Fig. 1(b), where the bold dashed line represents the wavelength of the excitation light, we observe that SPP can now be excited by a plane wave at normal incidence, since this dashed line intersects the dispersion curve at $q_{x}=0$.

In the case of an infinite grating, we compute the dispersion relation using the rigorous coupled wave analysis (RCWA) ${ }^{18-20}$ Figure 3 shows the normalized intensity of the electric field specularly reflected by an infinite grating of grooves engraved in a semi-infinite gold space in vacuum [Fig. 2(a)]. The reflection coefficient decreases when the plasmon mode is excited since most of the energy then goes into the SPP. The period is $L=600 \mathrm{~nm}$ and the width $a$ 

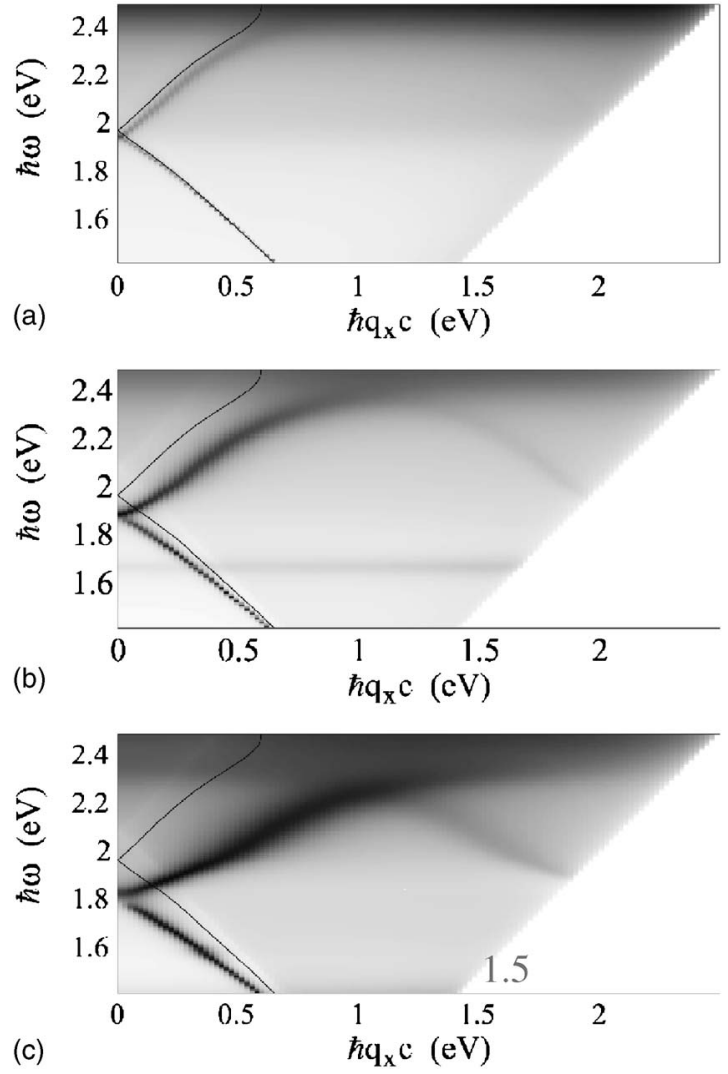

FIG. 3. Specular reflection coefficient of a plane wave incident on a grating engraved inside a semi-infinite gold space (low values in black). The periodicity is $600 \mathrm{~nm}$ and the width is $300 \mathrm{~nm}$ in the three cases. The depth of the grooves is: (a) $10 \mathrm{~nm}$, (b) $30 \mathrm{~nm}$, and (c) $50 \mathrm{~nm}$.

$=300 \mathrm{~nm}$ for the three plots in Fig. $3(\mathrm{dc}=0.5)$; three differ ent depths are investigated. The solid line in Fig. 3 corresponds to the periodically repeated dispersion curve of the free surface, Eq. (1). For a shallow modulation depth $(h$ $=10 \mathrm{~nm}$ ), the dispersion curve is only slightly modified with respect to the black line, see Fig. 3(a). The situation is rather different for the larger depths of 30 and $50 \mathrm{~nm}$, Figs. 3(b) and 3(c). More precisely, a normal incident field with energy $2.0 \mathrm{eV}(\lambda \approx 620 \mathrm{~nm})$ is able to excite the plasmon mode of the modulated metal-vacuum interface when the modulation depth is smaller than $10 \mathrm{~nm}$, but the coupling efficiency decreases a lot for larger values of $h$. In that case it is necessary to adapt the period and the width of the stripes in order to optimize the coupling at a fixed wavelength. For the geometries of Figs. 3(b) and 3(c), the period must be shorter than the plasmon wavelength. That result is in complete agreement with the calculations presented in Ref. 17. Finally we can notice in Fig. 3(c) that the width of the gap at the origin is very small, even for a depth of $50 \mathrm{~nm}$. This arises from the fact that this gap is formed by the interaction of two first order diffracted waves and it is a second order lift of degeneracy. ${ }^{17}$

Figure 4 shows the reflection coefficient for a plane wave at normal incidence on a $50 \mathrm{~nm}$ depth groove grating of varying period $L$ and width $a$. The dark vertical area corresponds to the excitation of the plasmon mode. We can see that the resonance condition does not depend much on the width $a$, but is very sensitive to the value of the period $L$. As

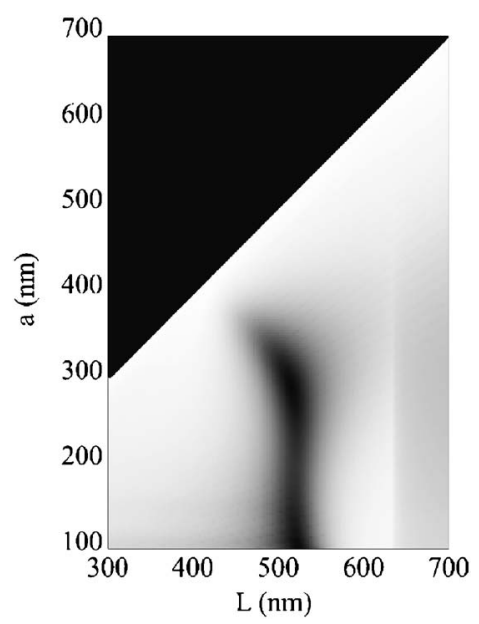

FIG. 4. Specular reflection coefficient of a plane wave incident on a groove grating engraved inside a semi-infinite gold space [high values (maximum of 1.0) in white, low values (minimum of 0.0 ) in black], with respect to the period $L$ and the width $a$ of the groove.

a matter of fact, the area where the specular reflection coefficient is minimum in Fig. 4 is elongated in the $a$ direction and very narrow in the $L$ direction. Actually, the optimal excitation is obtained for $a=290 \mathrm{~nm}$ and $L \approx 520 \mathrm{~nm}$, which is smaller than the free space SPP wavelength. Additionally, we can see that the value of the specularly reflected intensity decreases for $L>\lambda$, which is a consequence of the fact that the first diffraction order becomes radiative, opening a new loss channel.

\section{EFFECT OF FINITE SIZE}

In the following we consider finite gratings. Hence another parameter is the number of periods in the structure. It will be set to 5 in order to limit the number of degrees of freedom. Furthermore, the corresponding grating size is similar to that of the illumination spot in a realistic experiment. Because of the finite size, it becomes necessary to distinguish between two kinds of structures, as illustrated in Fig. 2. When gold is deposited on the interface, we will use the expression protrusions, Fig. 2(b). When matter is removed from the metal, we will as previously call it a groove grating, Fig. 2(a).

Two plasmonic systems are studied in this section. The first one is a semi-infinite metallic space, the second a symmetric metallic slab. The wavelength is fixed at $\lambda=633 \mathrm{~nm}$ and the grating illuminated with a monochromatic Gaussian beam at normal incidence, with a waist $w_{0}=6 \lambda$ corresponding to the length of the structure. The incident electric field is parallel to the $x$ axis.

\section{A. Semi-infinite gold space}

In order to evaluate the SPP excitation efficiency, we compute the intensity of the field just above the metallic surface $\left(z_{0}=0^{+}\right)$at $x_{0}=50 \mu \mathrm{m}$ away from the center of the grating (Fig. 2). This field intensity corresponds to that of the SPP traveling on the metal. Figure 5 shows the evolution of the intensity in $\left(x_{0}, z_{0}\right)$ for three different grating depths $h$, as a function of $L$ and $a$. We can see that the intensity is maxi- 

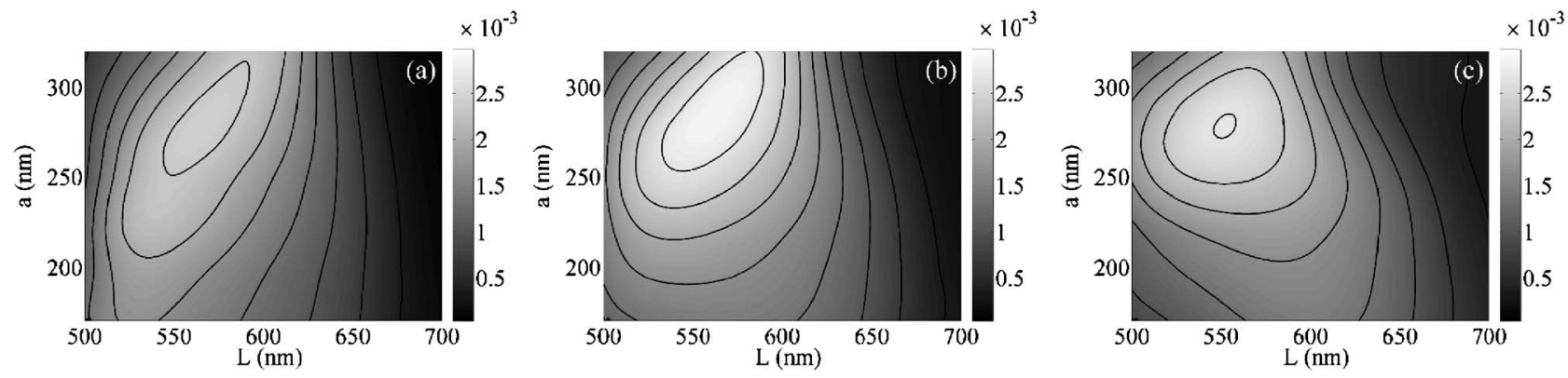

FIG. 5. Electric field intensity in $\left(x_{0}=50 \mu \mathrm{m}, z_{0}=0^{+}\right)$, normalized to the intensity of the incident field, as a function of the periodicity $L$ and the width of the defect $a$, for different heights $h$ of the modulated part: (a) $h=40 \mathrm{~nm}$, (b) $h=50 \mathrm{~nm}$, and (c) $h=60 \mathrm{~nm}$.

mum for $h=50 \mathrm{~nm}$. The corresponding period and width values are $L=550 \mathrm{~nm}$ and $a=280 \mathrm{~nm}$, which correspond to a duty cycle of almost $\mathrm{dc}=0.5$. The evolution of the maximal intensity and duty cycle are plotted in Fig. 6 as a function of the grating depth, for the groove grating. The difference between infinite and finite gratings can be attributed to the larger spectral width of the finite structure and to impedance mismatch at the transition between the modulated and the flat interfaces at the edges of the finite gratings.

Figure 7 compares the results obtained with symmetrical defects, i.e., an array of grooves, as in Fig. 5, and an array of protrusions. The results are perfectly identical in the case of an infinite grating excited by a plane wave when the transformation $L \rightarrow L, a \rightarrow L-a$ is applied to the groove data. This is not anymore the case for a finite grating because of the relative position of the protrusions/grooves with respect to
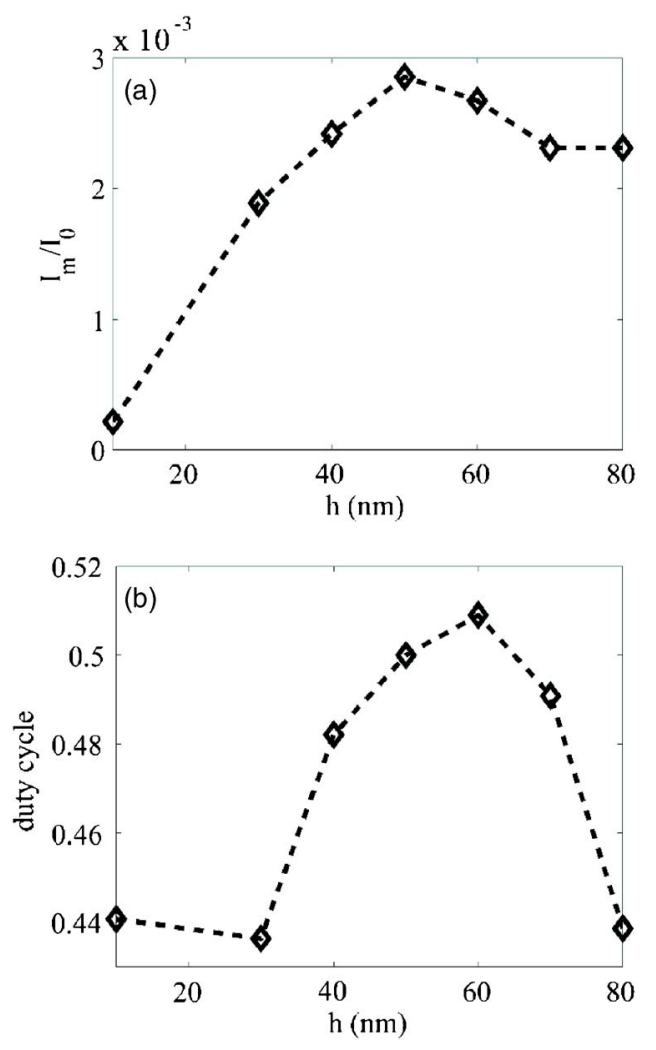

FIG. 6. (a) SPP intensity in $\left(x_{0}, z_{0}\right)$ for optimal period and width parameters, as a function of the height $h$. (b) Duty cycle for optimal coupling parameters as a function of $h$. Both curves correspond to a groove grating. the flat metal interface. Moreover, this symmetry occurs only in the case of the semi-infinite gold space and disappears with a finite thickness metallic film: in that last case, the average thickness is increased by a grating of protrusions and decreased by a grating of grooves.

In Fig. 7, panels (a) and (b) [(c) and (d)] show the results obtained with $h=50 \mathrm{~nm}(h=80 \mathrm{~nm})$. Figures 7 (a) and 7(c) correspond to a grating of grooves, and the quantity $L-a$ is reported on the vertical axis, which represents the width of the metallic part. Figures 7(b) and 7(d) correspond to a grating of protrusions plotted with respect to $L$ and $a$, the width of the metallic part. In the case of an infinite grating, these two configurations should be identical.

We can first note by comparing Figs. 7(a) and 7(b) that this correspondence is rather well verified for $h=50 \mathrm{~nm}$, the amplitudes and the positions of the maxima being quite similar. On the other hand the agreement is good only qualitatively for $h=80 \mathrm{~nm}$ [Figs. 7(c) and 7(d)]. Note that two maxima appear on these figures. The one with the largest period is located around $L=633 \mathrm{~nm}$, which corresponds to the illumination wavelength: just above this critical value, the first diffraction order which is fully evanescent for $L$ $<\lambda$ becomes radiative and diffracts parallel to the interface. Additional simulations have shown that the amplitude of this first diffraction order is very weak compared to the plasmon amplitude up to $h=50 \mathrm{~nm}$ in the case of protrusions and $h$ $=70 \mathrm{~nm}$ in the case of grooves. The fact that the first order seems to appear for lower values of $L$ than the wavelength is probably due to the finite size of the grating, which increases the number of wave vectors produced by the scattering of the incident field on the structure (in other words, the grating transmittivity is a broader band than it would be for an infinite grating).

\section{B. Symmetric gold film}

Let us now consider the symmetric gold film, where the metallic layer is embedded between two dielectric halfspaces. A SPP propagating in such a system does no longer obey the dispersion relation of Eq. (1). When the thickness of the film is similar to the penetration depth of the field in the metal, the SPP can feel the other surface and excite the corresponding wave. This coupling between the two interfaces produces two new modes. If the surrounding dielectric material is the same on each side, one mode is characterized by a symmetric parallel component of the electric field $E_{x}$ with 


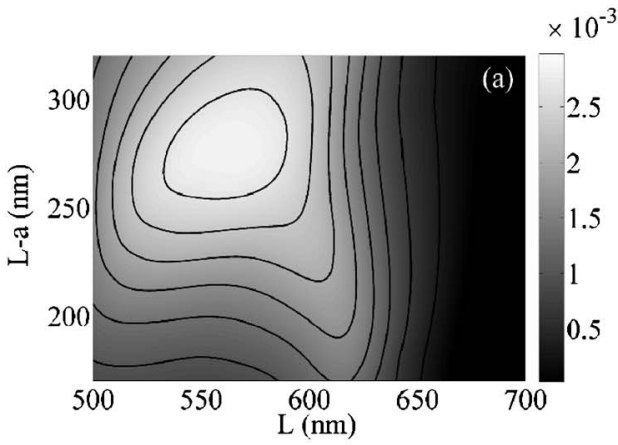

$$
\times 10^{-3}
$$

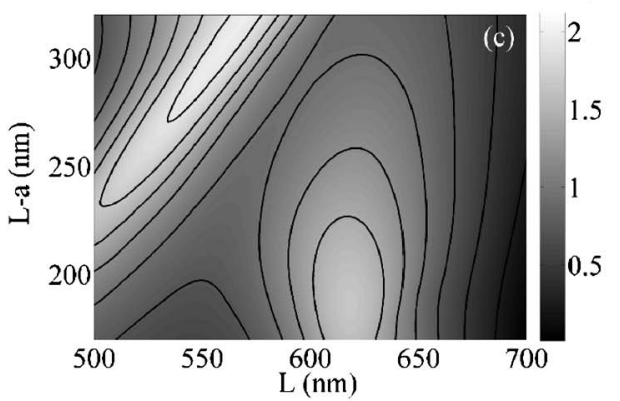

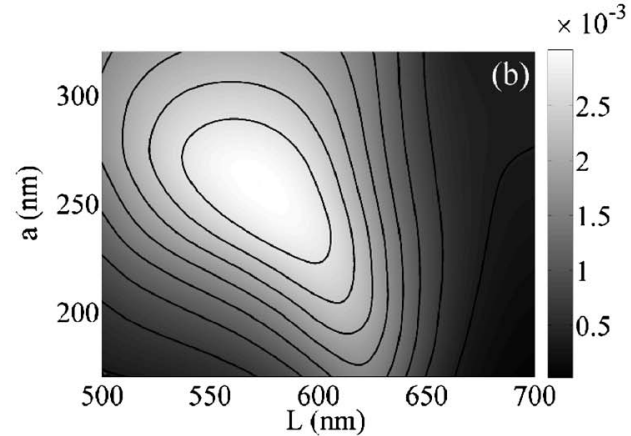

$\times 10^{-3}$

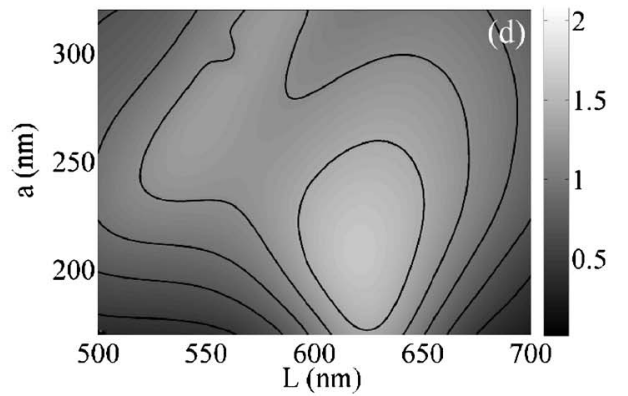

FIG. 7. SPP intensity in $\left(x_{0}, y_{0}\right)$ normalized to the intensity of the incident field, as a function of the period $L$ and the width $a$ of the defect. Two different grating heights are considered: [(a) and (b)] $h=50 \mathrm{~nm}$ and [(c) and (d)] $h$ $=80 \mathrm{~nm}$. Two types of gratings are investigated: [(a) and (c)] groove grating and $[(b)$ and (d)] protrusion grating. For comparison purpose, data for the groove grating are shown as a function of $L-a[(\mathrm{a})$ and (c)]. respect to the middle of the slab, while the other is antisymmetric. $^{21-24}$ This last one is the so-called long-range SPP, which propagates on the longest distance. For example, for a symmetric $70 \mathrm{~nm}$ thick gold slab at $\lambda=633 \mathrm{~nm}$ in air, the wavelength of the $E_{x}$-antisymmetric mode is $609 \mathrm{~nm}$, and its propagation length is $31.2 \mu \mathrm{m}$. For comparison, the $E_{x}$-symmetric mode has a wavelength of $580 \mathrm{~nm}$ and a range of $5.8 \mu \mathrm{m}$.

Figure 8 shows the normalized electric field intensity at the same location $\left(x_{0}, z_{0}\right)$ as a function of the parameters $L$ and $a$ for a five period grating deposited on a $70 \mathrm{~nm}$ thick gold layer surrounded on both sides by vacuum. Figures 8(a)-8(f) show the results for a protrusion (groove) grating.
The height of the defects is increased from $h=40 \mathrm{~nm}$ to $h$ $=60 \mathrm{~nm}$. In the case of protrusions, the best coupling efficiency is obtained for $h=50 \mathrm{~nm}, L=580 \mathrm{~nm}$, and $a$ $=270 \mathrm{~nm}$ which gives a duty cycle slightly smaller than dc $=0.5$. Additionally, as in Fig. 7(c), a second maximum appears when $h$ is larger than $60 \mathrm{~nm}$, which corresponds to the fact that the first diffraction order, which is then radiative, becomes sufficiently intense to dominate the SPP intensity. A comparison between the first row and the second row of Fig. 8 allows us to verify that the protrusion/groove symmetry does not occur for a finite thickness slab. Indeed, the best duty cycles are about $\mathrm{dc}=0.6$ for $h<60 \mathrm{~nm}$ and $\mathrm{dc}=0.4$ for a larger thickness. This effect is emphasized in Fig. 9. The
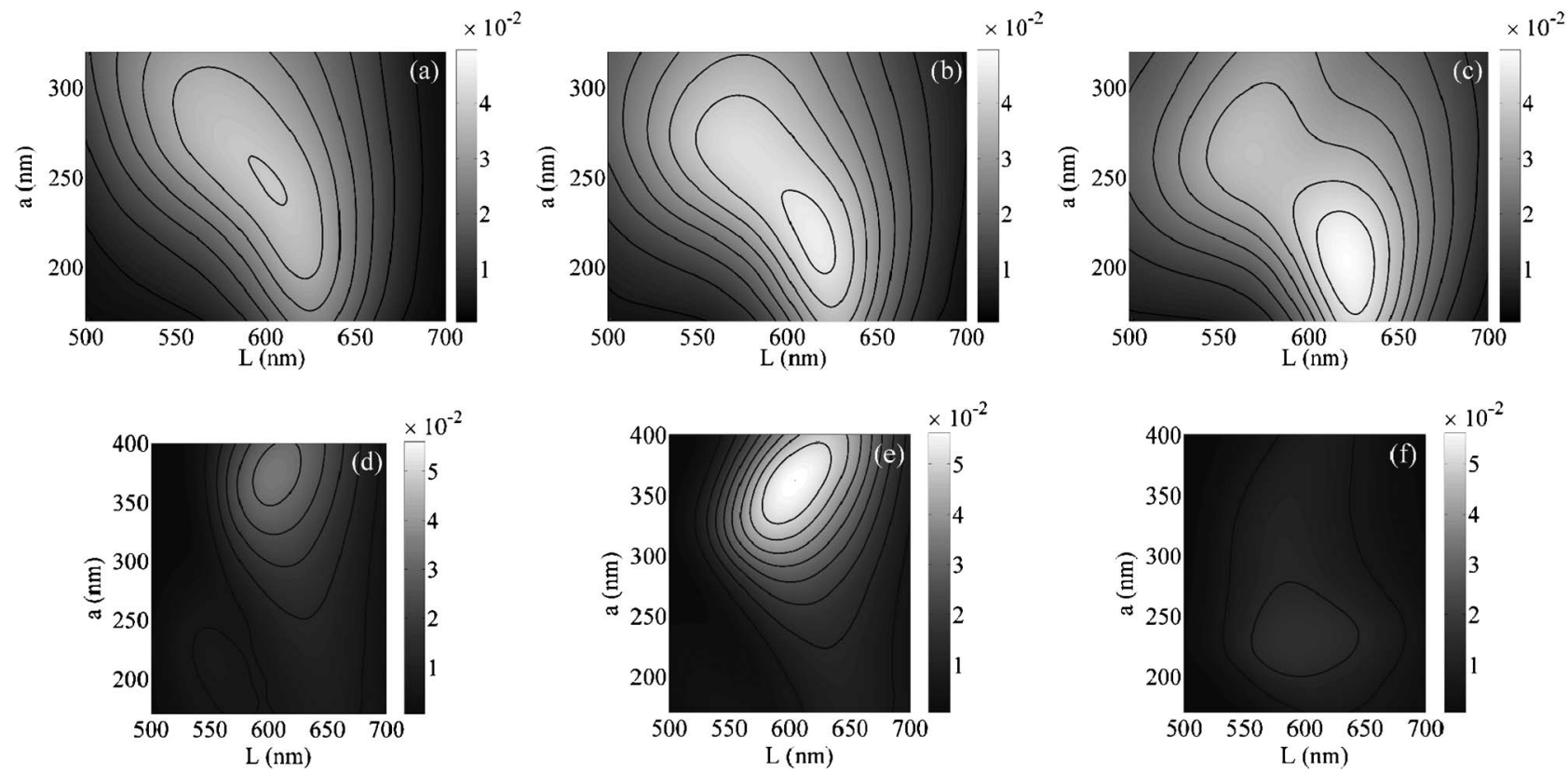

FIG. 8. SPP normalized intensity in $\left(x_{0}, z_{0}\right)$ for a grating on a $70 \mathrm{~nm}$ thick gold layer in air. [(a)-(c)] protrusion grating and [(d)-(f)] groove grating. Three different grating amplitudes are investigated: [(a) and (b) $] h=40 \mathrm{~nm},[(\mathrm{~b})$ and (e) $h=50 \mathrm{~nm},[(\mathrm{c})$ and (f) $] h=60 \mathrm{~nm}$. 

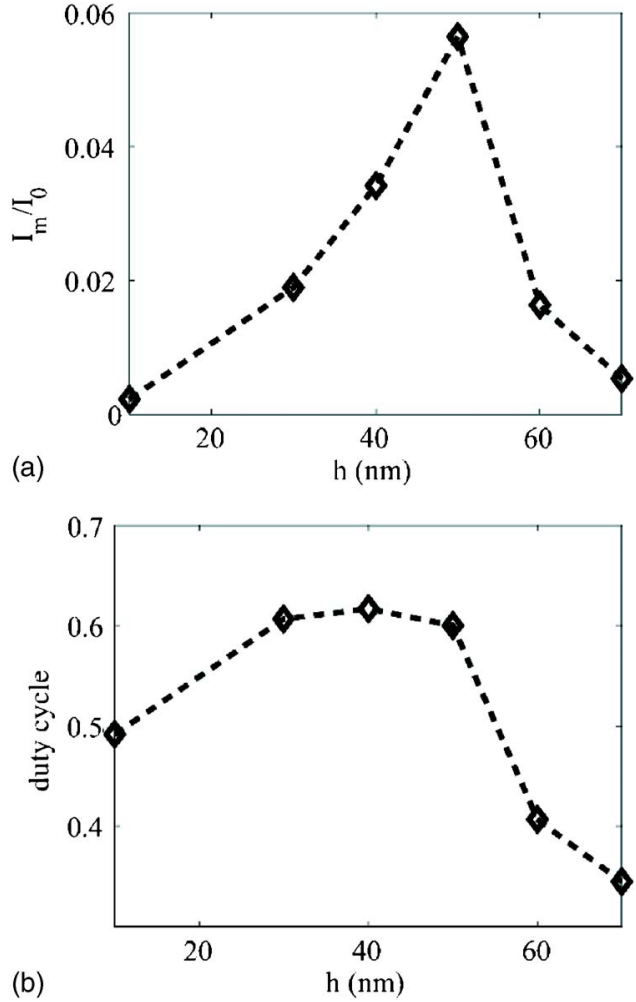

FIG. 9. (a) SPP intensity in $\left(x_{0}, y_{0}\right)$ for optimal grating period and width, as a function of the height $h$. (b) Duty cycle for best coupling parameters $(L$ and $h$ ) as a function of $h$. Both curves correspond to a groove grating on a gold slab.

optimal parameters for the groove configuration are $h$ $=50 \mathrm{~nm}, L=600 \mathrm{~nm}$, and $a=360 \mathrm{~nm}$. In this case the SPP intensity is larger than the optimized value for protrusion gratings. Hence a grating of grooves represents a more efficient way to excite a SPP than a protrusion grating.

In the two configurations discussed in this section, the semi-infinite gold space and the symmetric gold slab, the parameters allowing the best coupling efficiency correspond to a duty cycle between 0.5 and 0.6 and a modulation height of $h=50 \mathrm{~nm}$.

It is possible to evaluate the power transferred to the SPP by computing the norm of the difference between the incident Poynting vector and the transmitted one. We obtain a coupling efficiency of $45 \%$, that is, $22 \%$ in each direction in the case of the semi-infinite gold space and $33 \%$ (16\% in each direction) for the symmetric gold slab. These values are comparable to those obtained experimentally by Ditbacher $e t$ $a l$. for a gold slab on a silica substrate. ${ }^{11}$

\section{CONCLUSION}

We have presented a numerical investigation of the excitation of a SPP using finite gratings engraved in or deposited on a metal surface. Both a semi-infinite gold space and a symmetric gold slab surrounded by air have been investigated. The geometrical parameters of these finite gratings have been optimized to enhance the excitation of the surface plasmon. For both geometries, the optimal duty cycle is approximately $\mathrm{dc}=0.5$, the optimal modulation height $h$
$=50 \mathrm{~nm}$, and the best grating periodicity is smaller than the SPP wavelength on the corresponding flat interface. Furthermore, we have shown that there are only very little differences between an array of protrusions and an array of grooves in the case of a semi-infinite gold space, which corresponds to a rigorous property of an infinite grating illuminated by a plane wave. However, this symmetry is broken for a finite thickness slab due to the fact that the average thickness of the slab is larger for an array of protrusions than for an array of grooves: the most efficient coupling structure is a groove grating and the optimal duty cycles are about dc $=0.6$ if $h<60 \mathrm{~nm}$ and about dc=0.4 if $h>60 \mathrm{~nm}$.

As a conclusion, a grating of grooves is more efficient than a grating of protrusions. The period of this grating must be smaller than that of the SPP mode on the free interface. Additionally, there is no need for a large modulation depth of the grating, some tens of nanometers are sufficient, and the optimal duty cycle is generally around 0.5 . For these optimal parameters, the coupling efficiency to the SPP can reach $16 \%-22 \%$ in each direction, even with a grating as short as five periods.

\section{ACKNOWLEDGMENT}

Funding from the Network of Excellence Plasmo-NanoDevices (FP6-2002-IST-1-507879) is gratefully acknowledged.

${ }^{1}$ M. G. Cottam and D. R. Tilley, Introduction to Surface and Superlattice Excitations (Cambridge University Press, New York, 1989).

${ }^{2}$ P. Berini, R. Charbonneau, N. Lahoud, and G. Mattiussi, J. Appl. Phys. 98, 043109 (2005).

${ }^{3}$ J. Ditinger, S. Klein, F. Bustos, W. L. Barnes, and T. W. Ebbesen, Phys. Rev. B 71, 035424 (2005).

${ }^{4}$ F. Yu and W. Knoll, Anal. Chem. 76, 1971 (2004).

${ }^{5}$ N. Bassil, E. Maillart, M. Canva, Y. Lvya, M.-C. Millot, S. Pissard, R. Narwa, and M. Goossens, Sens. Actuators B 94, 313 (2003).

${ }^{6}$ A. Otto, Z. Phys. 216, 398 (1968).

${ }^{7}$ F. I. Baida, D. V. Labeke, and J. M. Vigoureux, Opt. Commun. 171, 317 (1999).

${ }^{8}$ S. I. Bozhevolnyi, V. S. Volkhov, A. Boltasseva, and K. Leosson, Opt. Commun. 223, 25 (2003).

${ }^{9}$ A. V. Zayats, I. Smolyaninov, and A. A. Maradudin, Phys. Rep. 408, 131 (2005).

${ }^{10}$ H. Ditlbacher, J. R. Krenn, G. Schider, A. Leitner, and F. R. Aussenegg, Appl. Phys. Lett. 81, 1762 (2002).

${ }^{11}$ H. Ditlbacher, J. R. Krenn, A. Hohenau, A. Leitner, and F. R. Aussenegg, Appl. Phys. Lett. 83, 3665 (2003).

${ }^{12}$ O. J. F. Martin and N. B. Piller, Phys. Rev. E 58, 3909 (1998).

${ }^{13}$ M. Paulus, P. Gay-Balmaz, and O. J. F. Martin, Phys. Rev. E 62, 5797 (2000).

${ }^{14}$ G. Lévêque, R. Mathevet, J. Weiner, G. C. des Francs, C. Girard, R. Quidant, J. C. Weeber, and A. Dereux, Nanotechnology 15, 1200 (2004).

${ }^{15}$ E. D. Palik, Handbook of Optical Constants of Solids (Academic, New York, 1985).

${ }^{16}$ N. W. Ashcroft and N. D. Mermin, Solid State Physics (HRW International Editions, Philadelphia, 1988).

${ }^{17}$ W. L. Barnes, T. W. Preist, S. C. Kitson, and J. R. Sambles, Phys. Rev. B 54, 6227 (1996).

${ }^{18}$ M. G. Moharam and T. K. Gaylord, J. Opt. Soc. Am. 73, 451 (1983).

${ }^{19}$ M. G. Moharam and T. K. Gaylord, J. Opt. Soc. Am. A 3, 1780 (1986).

${ }^{20} \mathrm{P}$. Lalanne and G. M. Morris, J. Opt. Soc. Am. A 13, 779 (1996).

${ }^{21}$ D. Sarid, Phys. Rev. Lett. 47, 1927 (1981).

${ }^{22}$ J. Burke and G. I. Stegeman, Phys. Rev. B 33, 5186 (1986).

${ }^{23}$ M. Fukui, V. C. Y. So, and R. Normandin, Phys. Status Solidi B 91, K61 (1979).

${ }^{24}$ E. N. Economou, Phys. Rev. 182, 539 (1969). 